\title{
Ultrasonic Measurements of Particle Concentration in a Multiphase Flow
}

\author{
Johan Carlson $^{\dagger}$ and Anders Grennberg ${ }^{\ddagger}$ \\ ${ }^{\dagger}$ Luleå University of Technology, Div. of Industrial Electronics, SE-971 87 Luleå, Sweden \\ ${ }^{\ddagger}$ Luleå University of Technology, Div. of Signal Processing, SE-971 87 Luleå, Sweden
}

\begin{abstract}
Non-invasive measurements of multiphase flows have several important applications in industry. In this paper we present a method that uses pulsed ultrasound and two small receivers to determine the mass concentration of iron ore particles in water.

The proposed method is based on the assumption that when ultrasound is transmitted through a scattering medium, the shape of the energy lobe changes. In this paper we use two receivers to monitor how the lobe changes.

We show with experiments how the proposed method can be used to determine particle mass fractions from 3 percent and up, with an accuracy of \pm 1 percent of the mass fraction. In the experiments we used a $3 \mathrm{MHz}$ transmitter and two receivers, one along the acoustical axis and the other $6 \mathrm{~mm}$ off-axis, to measure the mass fraction of a polydisperse suspension of iron ore powder in water.
\end{abstract}

\section{INTRODUCTION}

In several industries, such as the oil and gas, paper pulp, and mining industries, multiphase flows are common. It is often of great importance to be able to measure the mass or volume fraction of the different phases in such flows. In the mining industry, for example, iron ore powder is transported using water, and there is a need of measurement techniques to monitor the particle mass fraction. There exists several methods for doing this, all with their drawbacks and advantages. Some methods are based on optical techniques, which requires the flow to be transparent, other methods are based on nuclear magnetic resonance or inductance/conductance measurements. If the medium is opaque or if the solid particles are magnetic, these methods all have their drawbacks. Also, X-ray techniques and other methods based on radioactivity are both expensive and can be hazardous to the environment. A good overview can be found in the book by Chaoki, et al. [1], and in the review article by Whitaker [2].
The long term goal of our research project is to develop a ultrasonic technique for measuring mass fractions and mass fraction velocities in multiphase flows. The use of ultrasonic techniques has several advantages. It does not require the medium to be transparent. Depending on the frequency, it can be used to monitor both liquid/solid flows and liquid/gas flows.

In this paper we present a method that can be used to measure particle mass fractions in i multiphase flow consisting of water and iron ore particles. The method is based on pulsed ultrasound and the fact that transmitted pulse is scattered by the solid particles (see for example [3]). If we assume that the scattering results in a change of the shape of the lobe from the transmitting transducer, this change can be used to monitor concentration changes. If we calibrate the method for a given type of particles, the method can be used for online measurement of the particle mass fraction. The transmitted lobe can be measured by using an array of small receivers. In this paper we use only two receivers, and we show how the ratio of the energy received at the receivers can be used to estimate lobe changes.

\section{EXPERIMENTAL SetuP}

All experiments were conducted in the suspension container depicted in figure 2 . The bottom and the lid of the container is made of moulded plexiglass, which has acoustical characteristics similar to those of water. In order to reduce the influence of temperature fluctuations, the ambient temperature in the lab was controlled and lies within $\pm 1^{\circ}$ centigrade.

The transmitting ultrasound transducer, which had a center frequency of $3 \mathrm{MHz}$, was fixed to the bottom of the container, and the receivers ${ }^{1}$ were mounted on the top. One of the receivers was centered relative to the acoustical axis of the transmitter, and the other receiver was mounted $6 \mathrm{~mm}$ away from the center. The received signals were connected to a pre-amplifier and

\footnotetext{
${ }^{1}$ Panametrics XMS310 miniature immersion transducers
} 


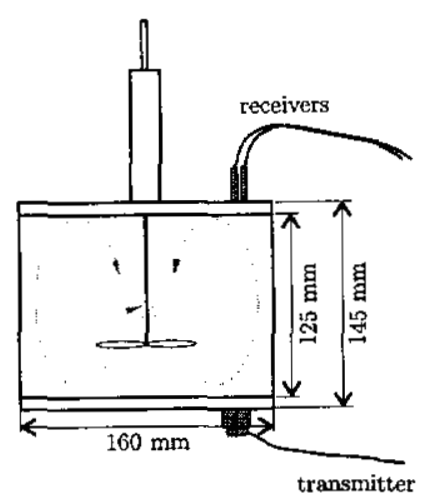

Fig. 1. Suspension container used in the experiments.

fed into a four-channel digitizing oscilloscope, with a sampling rate of $200 \mathrm{MHz}$.

All measurements were done on suspensions consisting of iron ore particles and water. The diameter of the particles varied from $0 \mu \mathrm{m}$ to $100 \mu \mathrm{m}$, which is much smaller than the wavelength of the sound. This means that the long wavelength limit is valid and that the scattering mechanisms derived from that are dominating (see for example [3]). Measurements were performed for mass fractions between 0 and 15 percent.

\section{THEORY}

\section{A. Principle}

Assume that the shape of the energy lobe from the transmitting transducer depends on the particle concentration. One way to measure the change in shape of the lobe is to use several receiving transducers. Figure 2 shows this for the case with one transmitter and two receivers, as used in our experiments.

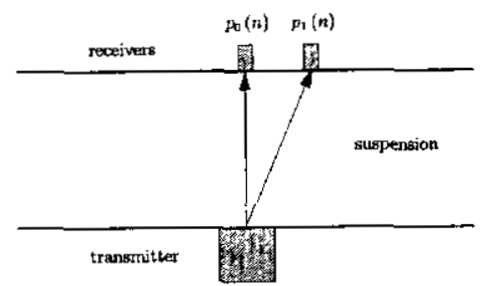

Fig. 2. Experimental setup with one transmitting transducer and two receivers.

Since both receivers simultaneously measure the same transmitted pulse, the received energy can be expected to be heavily dependent. It is therefore possible to compare the received pulses pair-wise, and then averaging over several measurements. A simple way to estimate the shape of the lobe is to calculate the received energy at each receiver. To determine how the transmitted energy spreads, we divide the received energy at receiver 1 with the received energy at receiver 0 , and then take the average of these ratios for $M$ subsequent measurements. We define the average energy ratio as

$$
E=\frac{1}{M} \sum_{m=1}^{M} \frac{E_{m, 1}}{E_{m, 0}}=\frac{1}{M} \sum_{m=1}^{M} \frac{\sum_{n=1}^{N} p_{m, 1}^{2}(n)}{\sum_{n=1}^{N} p_{m, 0}^{2}(n)}
$$

where $E_{m, 0}$ is the energy of $m:$ th received pulse at receiver 0 and $E_{m, 1}$ is the energy of $m:$ th received pulse at receiver 1 . Measurements indicate that for the concentration interval of interest, the ratio of energies depends on the particle mass fraction as

$$
E(c) \approx \alpha_{0}+\alpha_{1} c
$$

where $c$ is the mass fraction of particles and $\alpha_{0}$ and $\alpha_{1}$ are constants.

\section{B. Calibration}

For a specific system, with a given type of particles, the proposed method can be calibrated by making a least squares estimate of the parameters $\alpha_{0}$ and $\alpha_{1}$ in equation (2). To fit a straight line we need to measure and calculate the energy ratio for at least two different concentrations. We suggest calibrating at the lowest, and highest concentration of interest, at least. The need of calibration is a disadvantage, but as will be shown in the next section, the concentration interval for which the straight line approximation is valid is quite wide.

\section{On-line measurements}

Since the relationship between particle mass fraction and the energy ratio in equation (1) can be approximated by a straight line, measuring the mass fraction is done by calculating the energy ratio and solving the equation

$$
c=\frac{E(c)-\alpha_{0}}{\alpha_{1}} .
$$

\section{EXPERIMENTAL RESULTS}

In this section we describe the measurements made, and analyze the uncertainties involved in the mass fraction determination.

\section{A. Measurements}

As described in section II, measurements were made with mass fractions of iron ore particles from 0 to 15 
percent. For each particle concentration the average energy ratio for 100 received pulses was determined. The result is shown in figure 3 .

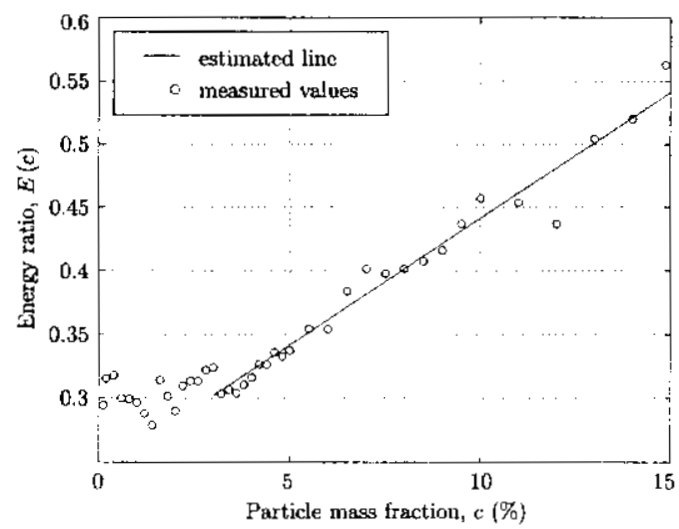

Fig. 3. Average energy ratio as a function of particle mass fraction in a suspension of iron ore and water. The estimated line was obtained using a least squares fit to the measured values.

In figure 3 we see that the approximation of a straight line is valid from about 3 percent and up. The straight line was estimated for this interval, and has equation

$$
E(c)=0.020+0.242 c
$$

We also note that the energy ratio $E$ increases with increased particle mass fraction. This indicates that the lobe from the transmitter is widened when concentration of scattering particles is increased.

For mass fractions of lower than three percent, the energy ratio in equation (1) did not show the same linear dependency on the mass fraction. Also, the variation in energy for the 100 measured pulses was larger for these concentrations (see figure 4). One explanation to this could be that the suspension is less homogeneous for lower mass fractions, resulting in larger variations in amplitude of the received pulses. Also, it might be the case that the dominating scattering mechanism changes at a certain concentration.

\section{B. Uncertainty analysis}

In this section we analyze the statistical properties of the proposed method, in order to estimate the uncertainty of the concentration measurements. Assume that the energy ratios of the received ultrasonic pulses can be regarded as random variables with the same, but unknown distribution. The central limit theorem [4] states that the average of several random variables from the same parent distribution will be more and

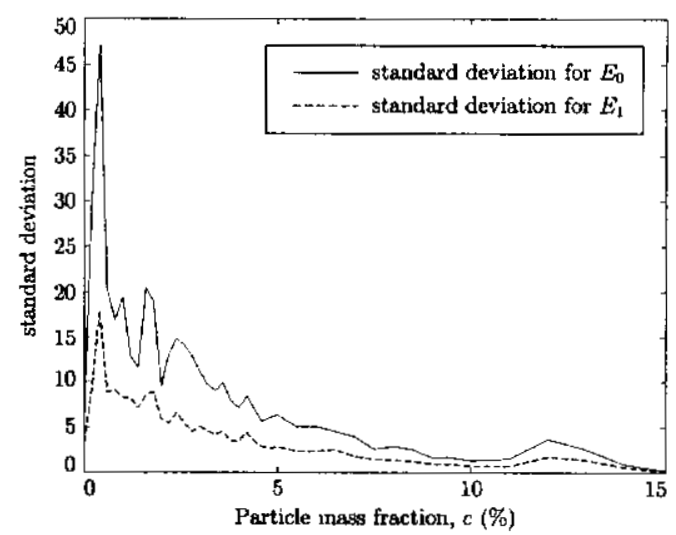

Fig. 4. Standard deviation of the energies, measured at receiver 0,1 respectively.

more normally distributed as the number of measurements increase. Thus, the average energy ratio $E$ in equation (1) can be assumed to be normally distributed. A $95 \%$ confidence interval can be determined for each concentration using equation (5) as

$$
E_{95}(c)=E(c) \multimap t_{95}(M-1) \widehat{\sigma}_{\text {ratio }},
$$

where $t_{95}(M-1)$ is the value of the $t$-distribution at $95 \%$ confidence level and $M-1$ degrees of freedom. In this case, where $M=100$, the $t_{95}$-value is approximately 1.98 . The confidence intervals are plotted in figure 5 .

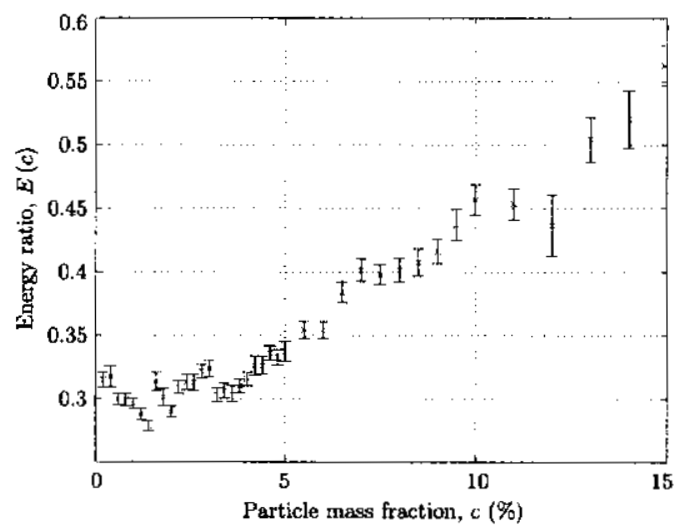

Fig. 5. Energy ratio as a function of particle mass fraction, with $95 \%$ confidence interval.

In figure 5 we see that the confidence interval becomes wider with larger mass fractions. This phenomenon can be explained by the fact that the signalto-noise ration (SNR) decreases as concentration increases. This is because the overall attenuation increases, causing the signal level to decrease. 
The uncertainty analysis indicates that it should be possible to measure the mass fractions in the interval 3 to 15 percent, with an accuracy of \pm 1 percent of the mass fraction. If the confidence interval becomes to wide, this is easily compensated for by increasing the number of measurements for those concentrations.

\section{CONCLUSIONS}

In this paper propose a simple and fast method that can be used to measure particle concentration in multiphase flows. This is illustrated with experiments with iron ore particles and water. The uncertainty analysis shows that it is possible to determine mass fractions with an error of less than $1 \%$ of the mass fraction. We have also showed that if we use two receivers, the average energy ratio in equation (1) depends linearly on the mass fraction in the range $3 \%$ to $15 \%$. This makes it easy to calibrate, and once the linear dependency has been determined, online measurements can be done, for a quite wide concentration interval.

For concentrations below three percent, the variation in pulse energies is very large and our method will not give accurate results.

We also see show the advantage of using two receivers is that we are able to block the measurements in pairs, which results in a significantly lower variation in the measured ratio than if the attenuation had been measured in only one point. This result should be possible to extend to more than two receivers, and will be one of the objectives of our future research.

If we combine the proposed method with some conventional transit-time bulk flow meter, for example the sing-around flow meter [6], this can lead to a method for measuring particle mass flows in multiphase flows.

\section{ACKNOWLEDGEMENTS}

The authors would like to express their gratitude towards Prof. Kerstin Vännman, Mr. Roger Öström and Mr. Svante Johansson for their valuable input on the uncertainty analysis. We would also like to thank Prof. Jerker Delsing for proof reading and for his valuable comments.

Generous grants from the Swedish Research Council for Engineering Sciences is also gratefully acknowledged.

\section{REFERENCES}

[1] Chaoki, J., Larachi, L., and Dudoković, M. P., Non-Invasive Monitoring of Multiphase Flows. Elsevier, 1997.

[2] Whitaker, T. S., "A Review of Multiphase Flowmeters and Future Development Potential," in Flow Measurement:
Proceedings of the 6th Int. Conf. on Flow Measurement FLOMEKO'93. (Seoul, Korea), pp. 628-634, Oct. 1993.

[3] Povey, M. J. W., Ultrosonic Techniques for Fluid Characterization. Academic Press, 1997.

(4] Box, G. E. P., Hunter, W. G., and Hunter, J. S., Statistics for Experimenters. John Wiley and Sons, 1978.

[5] Coleman, H. W. and Steele, W. G., Experimentation and Uncertainty Analysis for Engineers. John Wiley and Sons, 1989.

[6] Lynnworth, L. C., Ultrasonic Measurements for Process Control. Academic Press, 1989. 